\title{
Phase space density and chiral symmetry restoration in relativistic heavy ion collisions
}

\author{
Scott Pratt and Kevin Haglin* \\ Department of Physics and Astronomy and \\ National Superconducting Cyclotron Laboratory, \\ Michigan State University, East Lansing, MI 48824 USA
}

(September 13, 2018)

\begin{abstract}
The effect of altered hadron masses is studied for its effect with regard to final-state hadronic observables. It is shown that the final phase space densities of pions and kaons, which can be inferred experimentally, are sensitive to in-medium properties of the excited matter at earlier stages of the collision, but that the sensitivity is significantly moderated by interactions that change the effective numbers of pions and kaons during the latter part of the collision.
\end{abstract}

Relativistic heavy ion collisions at the CERN SPS $(160 A \mathrm{GeV} \mathrm{Pb}+\mathrm{Pb})$ or the Brookhaven AGS (11A GeV Au + $\mathrm{Au}$ ) produce a mesoscopic region where initial energy densities are in the neighborhood of a few $\mathrm{GeV} / \mathrm{fm}^{3}$ [迎2, several times the energy density of a proton. As the system expands and cools normal hadronic degrees of freedom become justified, but the system might still have novel properties when energy densities are in the region of $500 \mathrm{MeV} / \mathrm{fm}^{3}$. Most notably, as a consequence of restoring chiral symmetry, the masses of heavier hadrons might fall as much as $50 \%$ [3]. Evidence of falling masses has appeared in dilepton measurements of the $\rho$ peak in $\mathrm{Pb}+\mathrm{Pb}$ collisions at $E / A=160 \mathrm{GeV}$, performed at the SPS. The observed lack of strength of dilepton pairs in the region of the $\rho$ meson mass, combined with the observed additional strength for invariant masses near $400 \mathrm{MeV}$, suggest either that the $\rho$ meson was altered [4], dissolved [5], or broadened beyond recognition by collisions [6].

Since dileptons largely pass through the collision volume unscathed, they provide a transparent probe for investigating hadronic properties during the most interesting stages of the collision, but only those hadrons with the quantum numbers of the photon. Final state measurements of hadrons - pions, kaons, protons and hyperons, provide a rich chemistry as nearly $10^{3}$ hadrons are commonly produced in a single event. However, hadrons interact several times after the system has expanded beyond the energy densities of greatest interest when temperatures are near or above $150 \mathrm{MeV}$, and before the breakup density is reached, when temperatures are approximately $110 \mathrm{MeV}$. In the high density hadronic state, equilibrium chemical abundances can easily change by factors of two if masses are adjusted by several hundred $\mathrm{MeV}$ as predicted by some chiral models. In this letter we investigate whether such mass changes, and the corresponding changes in hadronic chemical abundances, survive and produce a signal in the measured hadrons. By using phenomenologically motivated chemical rates, we model the chemical development of a kinetically equilibrated gas that expands under a time scale relevant for relativistic heavy ion collisions. We find that falling hadron masses, on the order of 50\%, result in increased final phase space densities for both pions and kaons which can be inferred by measuring spectra and two-particle correlations. We show that if reactions that preserve the effective pion and kaon numbers are ignored, a strong signal survives the final expansion, and that if such reactions are included, the manifestations of the original novel chemistry survive, but are strongly moderated. We also find that hadronic observables are sensitive to the issue of whether both baryon and vector mesons scale, or whether just the baryon masses [7]:8].

In $\mathrm{Pb}+\mathrm{Pb}$ collisions at the SPS or in $\mathrm{Au}+\mathrm{Au}$ collisions at the AGS, $\approx 7 \mathrm{fm} / \mathrm{c}$ after initial contact, matter has expanded and cooled to the point where a model based on binary interactions of hadrons is justified. For the following thermal calculations, we take the onset of binary modeling to occur at a temperature of $160 \mathrm{MeV}$. We assume that the system is both kinetically and chemically equilibrated at this point and is characterized by four numbers: a baryon chemical potential $\mu_{b}$, a strangeness chemical potential $\mu_{s}$, and a temperature $T$. The strangeness chemical potential is chosen such that the net strangeness is zero. It would be zero if the baryon density were zero. The baryon chemical potential is chosen to match the effective baryon to pion ratios, unity for the AGS example and $1 / 5$ for the SPS example. The assumption of equilibration when $T=160 \mathrm{MeV}$ is an ansatz, justified only by the fact that the hadrons significantly overlap at higher temperature, suggesting very rapid equilibration of the strongly interacting system. At later times and lower temperatures, kinetic equilibrium is approximately maintained [9], but some aspects of chemical equilibrium are lost. When the system expands and cools to approximately $T=110 \mathrm{MeV}$, kinetic equilibrium is lost

\footnotetext{
${ }^{*}$ Current address: Department of Physics, Astronomy and Engineering Science, St. Cloud State University, St. Cloud, MN 56301
} 
as well and the system dissolves.

As the system expands, the effective numbers of kaons and pions must adjust to the rapidly changing environment. The effective number of pions is defined as the number of pions plus the sum of other hadrons weighted by their effective pionic content. For instance, a $\rho$ meson counts as two pions since it decays principally into two pions, while the effective pionic content of a $\Delta$ baryon is one. Reactions such as $\rho \leftrightarrow \pi \pi$ are rapid and can keep the number of $\rho$ mesons in equilibrium but do not change the overall effective pion number [10,11]. Reactions that change the overall pion number, such as $\pi \pi \leftrightarrow \rho \rho$, are not sufficiently fast and allow the system to lose chemical equilibrium. One can characterize the unequilibrated state by an effective chemical potential $\mu_{\pi}$ that corresponds to the "conserved" pion number. One can similarly assign an effective chemical potential to the effective kaon number. Although the net strangeness is zero, the net number of strange quarks must adjust during the expansion. Reactions that conserve the net number of strange quarks occur rapidly, e.g. $K^{-} p \leftrightarrow \Lambda \pi$, but reactions that change the number of strange quarks, e.g. $\pi \pi \leftrightarrow K K$, are slow as they require a strange and an anti-strange hadron to either interact or be be produced jointly. We again describe this lack of chemical equilibrium via an effective chemical potential $\mu_{K}$ [12] that corresponds to the effective kaon number, where a kaon or $\Lambda$ baryon counts as one kaon as they each have one strange quark. Either an $\Omega$ baryon (with quark content sss) or an $\bar{\Omega}$ would count as three kaons. Both $\mu_{K}$ and $\mu_{\pi}$ become zero in chemically equilibrated systems.

When a system is perturbed from chemical equilibrium, $\mu_{K}$ and $\mu_{\pi}$ approach equilibrium exponentially with characteristic times $\tau_{K}$ and $\tau_{\pi}$. These times have been studied by Song and Koch for a simple meson gas [10]. Our analysis includes a greater variety of hadrons. We include the spin $1 / 2$ baryon octet, the baryon spin $3 / 2$ dectet, the pseudoscalar meson nonet and the vector meson nonet, but not those resonances which correspond to orbital excitations of mesons in the framework of the constituent quark model. Given the cross sections for creating pions in individual reactions, we estimate the time reversed rate through time-reversal arguments.

$$
\begin{aligned}
\frac{d N_{\pi}}{d^{3} x d t} & =\sum_{i} \Delta N_{i, \pi} R_{i}\left(1-\exp -\Delta N_{i, \pi} \mu_{p} i\right) \\
R_{i} & =\frac{\left(2 J_{a}+1\right)\left(2 J_{b}+1\right)}{(2 \pi)^{6}} \int \frac{E_{c m} d^{3} P}{E} e^{\left.-\left[E-\left(N_{a, \pi}+N_{b, \pi}\right) \mu_{\pi}\right)\right] / T} \int d^{3} q \sigma\left(E_{c m}\right) v_{r e l, c m}
\end{aligned}
$$

where the sum is over all reactions $(a b \rightarrow X)$ that increase the effective pion number by $\Delta N_{i, \pi}$. The effective rate per volume, $R_{i}$, for a specific reaction can be found by convoluting the phase space densities of $a$ and $b$, and folding in the cross section. The energy of the pair is $E=\sqrt{P^{2}+E_{c m}^{2}}$, where $E_{c m}$ is the energy of the pair as measured in the $a b$ rest frame and $q$ and $v_{r e l, v m}$ are the relative momentum and relative velocity of $a$ and $b$ as measured in that frame. The baryon and strangeness chemical potentials have been omitted for brevity. The integral over $P$ can be performed analytically, and given an expression for the cross section, the integral over $q$ can be performed numerically.

The effective pion density,

$$
n_{\pi}=\sum_{a} N_{a, \pi}\left(2 J_{a}+1\right) \int \frac{d^{3} p}{(2 \pi)^{3}} e^{-\left(E-\mu_{\pi} N_{a, \pi}\right)},
$$

can also be found analytically given the effective pionic content, $N_{a, \pi}$ of the species $a$. One can expand the above expressions for small $\mu_{\pi}$ to find the rate at which $\mu_{\pi}$ returns to equilibrium,

$$
\begin{aligned}
\frac{d \mu_{\pi}}{d t} & =-\frac{1}{\tau} \mu_{\pi} \\
\frac{1}{\tau} & =\frac{\sum_{i} \Delta N_{i, \pi}^{2} R_{i}\left(\mu_{\pi}=0\right)}{n_{\pi}\left(\mu_{\pi}=0\right)}
\end{aligned}
$$

Unfortunately, experimental cross sections are not available for the majority of the combinations $a b$ of the 26 mass states used in this analysis, and since the relevant energies for pion production are several hundred MeV, perturbation theory is not particularly reliable. We have therefore instituted simplified expressions for the cross sections.

$$
\sigma\left(E_{c m}\right)=\sigma_{0} \theta\left(E_{c m}-E_{t h}-N_{\pi, X} 350 \mathrm{MeV}\right),
$$

where $N_{\pi, X}$ is the number of pions in the final state, and the threshold $E_{t h}$ is the minimum energy of a state with the quantum numbers of the initial state. This behavior is motivated by the observed behavior of the inelastic $p p$ and $p \pi$ cross sections 13] with $\sigma_{0}$ being $25 \mathrm{mb}$ if both incoming particles are baryons, $20 \mathrm{mb}$ if one is a baryon and 15 $\mathrm{mb}$ if neither is a baryon. When the production of more pions becomes available, all the cross section is then devoted to that number of pions. For the case where no baryons are present in the initial state, only even numbers of pions 
are allowed in the final state as required by conservation of g-parity. For the production of strangeness, only pairs of strange-anti strange are allowed and the steps are in units of $1.2 \mathrm{GeV}$ if no baryons are present in the initial state and $800 \mathrm{MeV}$ if baryons are present.

$$
\sigma\left(E_{c m}\right)=\sigma_{0} \theta\left(E_{c m}-E_{t h}-N_{K, X}(800 \text { or } 1200 \mathrm{MeV})\right),
$$

The reduced stepsize of $800 \mathrm{MeV}$ is to account for $\Lambda$ production which only requires a few hundred $\mathrm{MeV}$ energy. The cross section $\sigma_{0}$ for strangeness production is taken as one fourth that of the pion production cross section. These values were motivated by measurements of $\Lambda$ production [14,15], with the value of $\sigma_{0}$ being multiplied by 2.5 to account for the production of other hyperons. Although the prescription for determining cross sections is phenomenologically motivated, given the large number of experimentally unknown information, the cross sections must not be taken seriously beyond the $50 \%$ level, especially for the case of strangeness producing rates.

Characteristic chemical equilibration times are displayed in Figure 1. Characteristic expansion times are $\approx 5 \mathrm{fm} / \mathrm{c}$ when $T=160 \mathrm{MeV}$ and $\approx 20 \mathrm{fm} / \mathrm{c}$ when the system is near breakup at $T \approx 110 \mathrm{MeV}$. From viewing Figure 1 one expects pionic chemical equilibrium to be lost when the temperature approaches $150 \mathrm{MeV}$ while strangeness equilibration can be hardly justified even at $T=160 \mathrm{MeV}$. We should point out that if masses fall due to restoration of chiral symmetry, hadron densities rise accordingly and characteristic times are considerably shorter. The role of the baryons in maintaining chemical equilibrium is crucial. Even when baryons comprise only a fifth of the produced hadrons, reactions involving baryons contribute the majority of the rate. This comes from the fact that reactions involving baryons do not need to conserve g-parity and pions need not be produced pairwise.

We now proceed to calculate generated chemical potentials for systems which cool from an equilibrated state at $T=160 \mathrm{MeV}$ to an unequilibrated state at $T=110 \mathrm{MeV}$ with chemical potentials $\mu_{\pi}$ and $\mu_{K}$. If number-changing rates are neglected the four final chemical potentials, $\mu_{\pi}, \mu_{K}, \mu_{s}$ and $\mu_{b}$ can be found by the four constraints: (1) The net strangeness is zero. (2) The baryon to pion ratio is fixed. (3) The entropy per pion is fixed. (4) The net number of strange quarks per pion is fixed. If rates that change the pion number and strange quark number are included, the latter three constraints must be modified by integrating the time development of the system.

$$
\begin{aligned}
\frac{d}{d t} \frac{n_{b}}{n_{\pi}} & =-\frac{n_{b}}{n_{\pi}^{2}} \frac{d}{d t} n_{\pi} \\
\frac{d}{d t} \frac{n_{S}}{n_{\pi}} & =-\frac{n_{S}}{n_{\pi}^{2}} \frac{d}{d t} n_{\pi}-\frac{\mu_{\pi}}{n_{\pi}} \frac{d}{d t} n_{\pi}-\frac{\mu_{K}}{n_{\pi}} \frac{d}{d t} n_{K} \\
\frac{d}{d t} \frac{n_{K}}{n_{\pi}} & =-\frac{n_{K}}{n_{\pi}^{2}} \frac{d}{d t} n_{\pi}+\frac{1}{n_{\pi}} \frac{d}{d t} n_{K},
\end{aligned}
$$

where $n_{S}$ is the entropy density. Thus if one knows the temperature as a function of time, one can integrate these equations forward in time, using the four aforementioned quantities to determine the four unknown chemical potentials at any time. For our purposes we assumed a simplified behavior of the temperature, $T$, as a function of time, $d T / d t$ $=-6.5 \mathrm{MeV} /(\mathrm{fm} / \mathrm{c})$, which was motivated by the behavior observed in cascade simulations [9].

The resulting evolutions of $\mu_{\pi}$ and $\mu_{K}$ as a function of temperature are illustrated in Figure 2. The baryon to pion ratio was chosen to be 0.2 , which is relevant for $\mathrm{Pb}+\mathrm{Pb}$ collisions at the SPS. Inclusion of the rates is clearly important in determining the final chemistry.

The chemical evolutions were also calculated with the assumption that the hadronic masses varied as a function of the temperature. The hadrons, aside from the pseudoscalar mesons which are Goldstone bosons, were assumed to scale linearly with the temperature from their vacuum mass when $T=110 \mathrm{MeV}$ to a fraction, $m / m_{0}$, at $T=160$ $\mathrm{MeV}$. The lower panels of Figure 3 show the value of $\mu_{\pi}$ at breakup for four cases. The final baryon to pion ratio was chosen to be 0.2 or 1.0, roughly appropriate for SPS or AGS conditions respectively. If no number-changing rates are included, the generated chemical potential is large, approaching $100 \mathrm{MeV}$ for the SPS case and $150 \mathrm{MeV}$ for the AGS case, when the mass reduction factor falls below 0.5. However, inclusion of the rates reduces the resulting chemical potential to near $50 \mathrm{MeV}$. Since the falling of the $\rho$ mass is controversial, the calculations were repeated with the assumption that only the baryon masses scaled (right panels of Figure 3), while the vector meson masses remained fixed. In this case the resulting chemical potentials were far lower for the SPS case, and in fact chemical potentials were smaller for increasingly small mass reduction factors. This owes itself to the fact that the entropy per pion due to the presence of baryons is rather high, compared to the entropy per pion in the mesonic sector.

Calculations of $\mu_{K}$ as a function of the mass reduction factor are displayed in the upper panels of Figure 3 . The resulting $\mu_{K}$ is much larger than $\mu_{\pi}$, surpassing $100 \mathrm{MeV}$, even for the case where rates were included. Since the phase space density is proportional to $e^{\mu / T}$, the strangeness phase space density is nearly doubled compared to the $\mu_{K}=0$ case if masses don't scale, and more than doubled when mass-scaling occurs. The number-changing rates that most strongly affected $\mu_{K}$ were not those that changed the effective kaon number but those that affected the net 
pion number. The kaon-number-changing rates are sufficiently small that they had a relatively small effect toward the final outcome.

Chemical potentials can be inferred from hadronic measurements via correlation measurements [16]. Combining two-particle correlations measurements, which are sensitive to the breakup volume, and spectra one can infer phase space densities, which should be $\approx e^{\mu / T}$, at low momentum. Combined with careful modeling of the breakup stage of the reaction, one can thus infer chemical potentials to the accuracy of $\pm 30 \mathrm{MeV}$.

Perhaps the observable that is most directly sensitive to $\mu_{K}$ is the abundance of anti-hyperons. For instance, $\bar{\Omega}$ consists of three anti-quarks, and would feel an enhancement of $\exp \left(3 \mu_{K} / T\right)$. Figure 4 displays the ratio $\bar{\Lambda} / \bar{p}$ as a function of the mass reduction factor. The baryon-to-pion ratio was set to 0.2 or 1.0 to be relevant for the SPS and AGS experiments respectively. Calculations where number changing rates are included or not included are both shown, and it is evident that number-changing rates have a profound influence on the population of anti-hyperons. When rates are included, there is little sensitivity to the mass reduction factor, whereas when number changing rates are ignored, calculations yield very large ratios. Preliminary measurements of the $\bar{\Lambda} / \bar{p}$ by E864 [17 have yielded values above unity, approximately double or triple the calculations presented here. If one argued that the cross sections for number changing rates used here, particularly those that changed the effective number of strange quarks, were overestimated by a factor of two, one could then explain the experimental findings. Before one can make firm conclusions with regard to this measurement, a better understanding of number-changing cross sections, especially those involving the annihilation of strange hyperons, must be reached.

Several conclusions can be made from this investigation. First, the chemical properties of the hottest stages of relativistic heavy ion collisions do indeed manifest themselves in the final hadronic measurements. In particular, chiral restoration can affect the final phase space densities of both the pions and kaons. However, chemical processes, which drive the system towards equilibrium, significantly mitigate such signals. Altering the chiral properties of the hottest stages results in a difference of the final chemical potentials of the order of $50 \mathrm{MeV}$ or less, while the ability of an experiment to determine the chemical potentials, is probably in the $30 \mathrm{MeV}$ range at best. Thus, measuring hadrons can be expected to provide information or evidence regarding the chiral transition, but would probably not supply a "smoking gun" signal, unless the chemical rates employed in this paper are overestimated.

It should be stressed that this analysis has relevance beyond the issue of dropping hadron masses. Effective chemical potentials and phase space densities are of crucial importance in understanding the entropy of the reaction, which can signal the presence of the deconfinement transition. Secondly, the conditions of the final state provide boundary conditions necessary for modeling the earlier stages of the collision. For instance, dilepton production from $\pi \pi$ annihilation would scale as $\exp \left(2 \mu_{\pi} / T\right)$. Finally, the sensitivity of the observables to the inclusion of pion-numberchanging and kaon-number-changing chemical processes demonstrates the importance of including such reactions, along with the corresponding time-reversed processes, in any modeling of the final state expansion.

\section{ACKNOWLEDGMENTS}

The authors thank Yang Pang for pointing out the importance of baryons in hadronic chemistry. This work was supported by the National Science Foundation under grant PHY-9605207.

[1] T. Peitzmann, et al., Nucl. Phys. A610, 200c (1996).

[2] S. Margetis, et al., Nucl. Phys. A590, 355c (1995).

[3] G.E. Brown and M. Rho, Phys. Rev. Lett. 66, 272 (1991).

[4] G.Q. Li, C.M. Ko, and G.E. Brown, Phys. Rev. Lett. 75, 4007 (1995); G.Q. Li, C.M. Ko, and G.E. Brown, Nucl. Phys. A606, 503 (1996).

[5] P.J. Siemens and S.A. Chin, Phys. Rev. Lett. 55, 1266 (1985).

[6] G. Chanfray, R. Rapp and J. Wambach, Phys. Rev. Lett. 76, 368 (1996).

[7] R. Pisarski, Phys. Rev. D52, R3773 (1995).

[8] V. Koch, Intl. Jour. Mod. Phys. E (1997).

[9] S. Pratt and J. Murray, Phys. Rev. C57, 1907 (1998).

[10] C. Song and V. Koch, Phys. Rev. C55, 3026 (1997).

[11] S. Gavin and V. Ruuskanen, Phys. Lett. B262, 326 (1991); S. Gavin, Nucl. Phys. B351, 561 (1991).

[12] J. Rafelski, Acta Physica Polonica 27, 1037 (1996). 
[13] Particle Data Group, The 1998 Review of Particle Properties, www-pdg.lbl.gov.

[14] A. Sibirtsev, Phys. Lett. B359, 29 (1995).

[15] V. Flaminio, et al.., CERN preprint CERN-HERA 01/84 (1984).

[16] G.F. Bertsch, Phys. Rev. Lett. 72, 2349 (1994).

[17] T.A. Armstrong, et al., Phys. Rev. Lett. 79, 3351 (1997). 


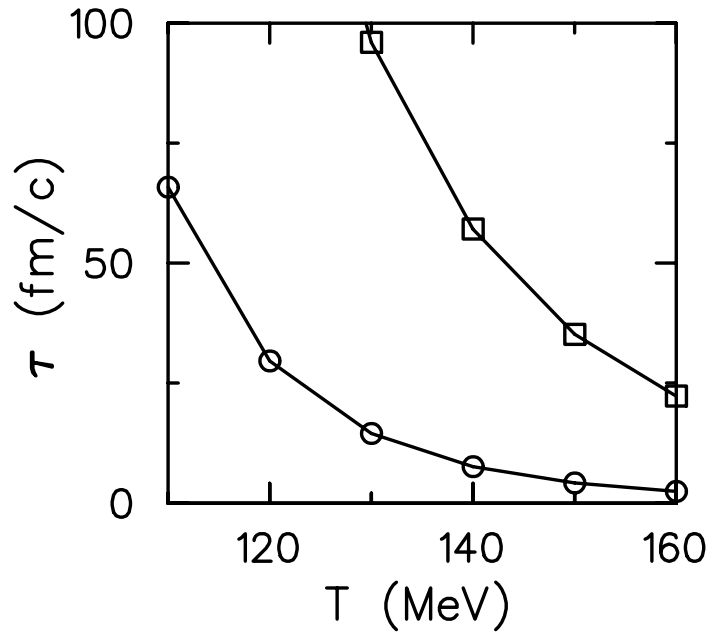

FIG. 1. Characteristic times for chemical potentials returning to zero are shown for $\mu_{\pi}$ (circles) and $\mu_{K}$ (squares). The decay time corresponding to $\mu_{\pi}$ becomes larger than characteristic expansion times when $T \approx 150 \mathrm{MeV}$, while equilibration times for $\mu_{K}$ are longer than characteristic expansion times even for $T=160 \mathrm{MeV}$.

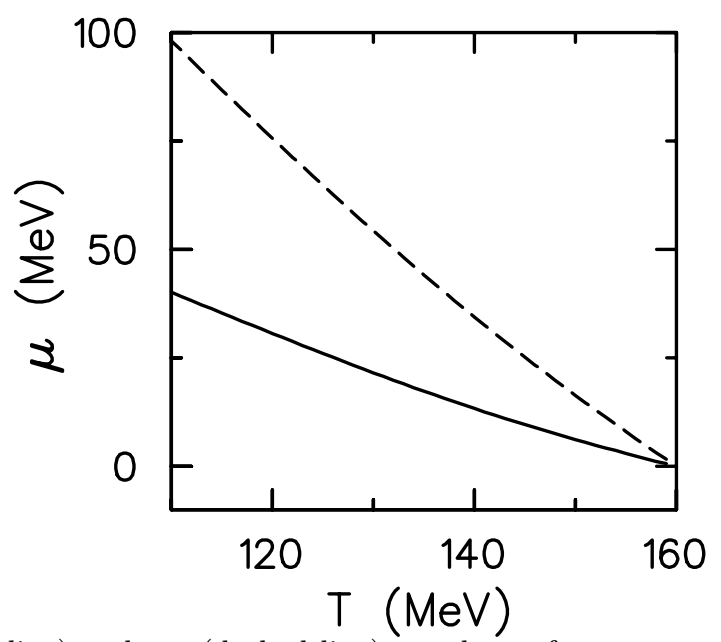

FIG. 2. The evolution of $\mu_{\pi}$ (solid line) and $\mu_{K}$ (dashed line) are shown for an expanding hadron gas that began at a 160 $\mathrm{MeV}$ temperature. This calculation has incorporated pion and kaon number changing rates. 


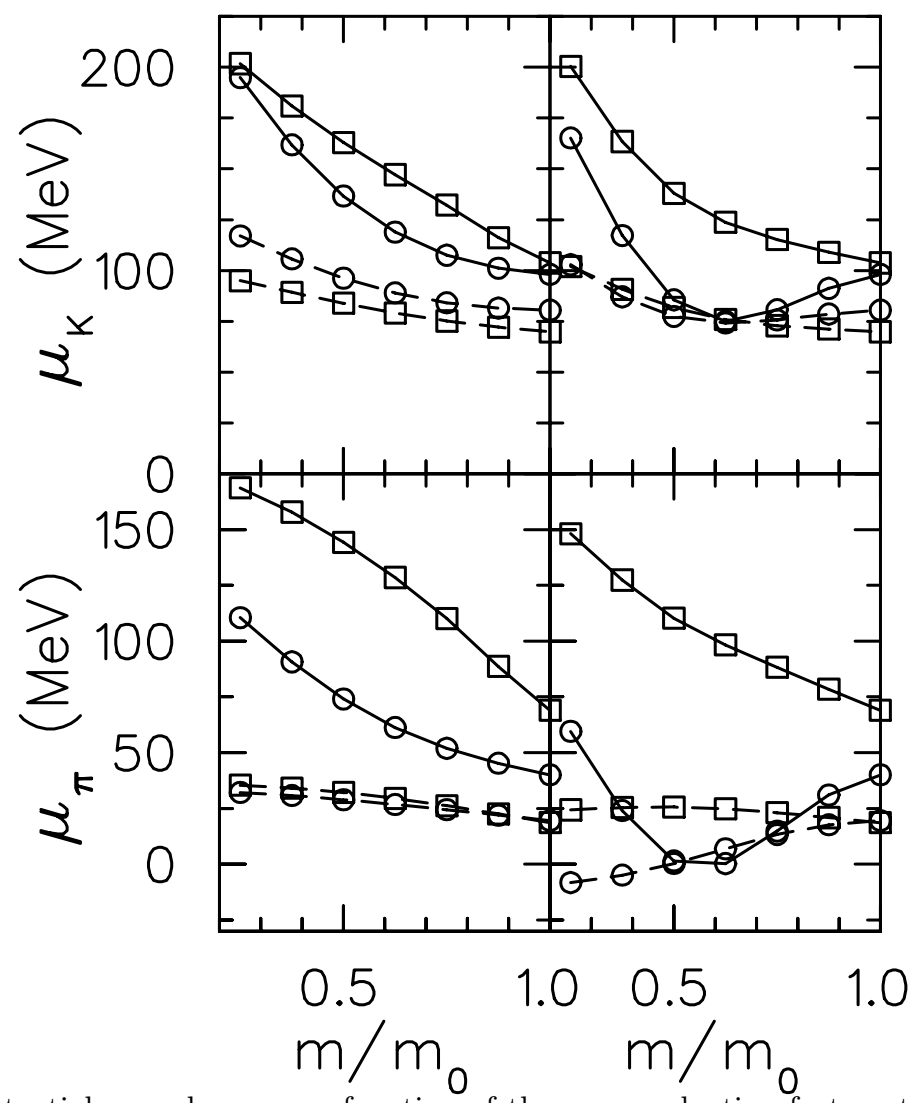

FIG. 3. Final chemical potentials are shown as a function of the mass reduction factor at the initial temperature of 160 $\mathrm{MeV}$. Calculations are shown for the cases where number-changing processes are neglected (solid lines) or included (dashed lines), and for when vector mesons scale with the baryon masses (left panel) or remain fixed (right panel). Calculations are performed for two choices of the baryon-to-pion ratio, 1.0 which is relevant for AGS measurements (squares) and 0.2 which is relevant for measurements at SPS (circles). One sees that number-changing rates significantly damp the effect of altering hadron masses, and if vector meson masses are unchanged that the chemistry is less affected.

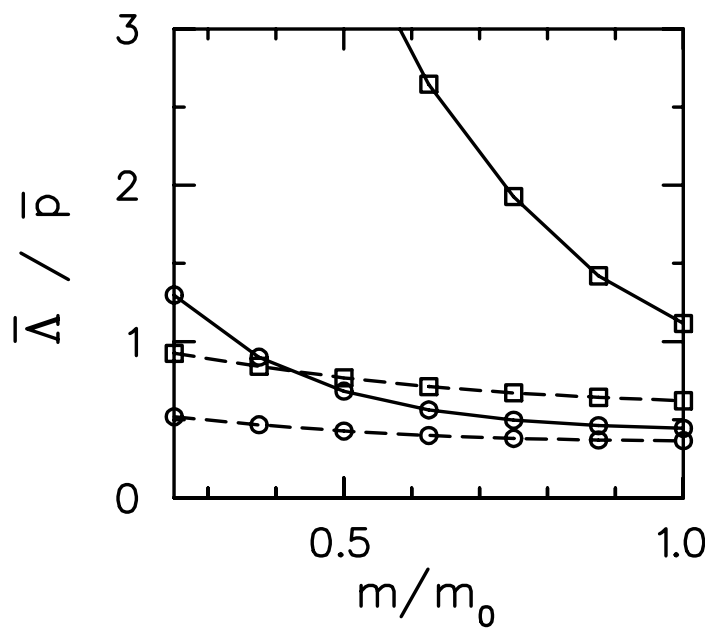

FIG. 4. The $\bar{\Lambda} / \bar{p}$ ratio is plotted as a function of the mass reduction factor. Calculations are shown for the cases where number-changing processes are neglected (solid lines) or included (dashed lines). Calculations are performed for the two choices of the baryon-to-pion ration, 1.0 which is relevant for AGS measurements (squares) and 0.2 which is relevant for measurements at CERN (circles). Calculations are sensitive to both the inclusion of number-changing rates and alterations of hadronic masses. 